\title{
Large deflection of clamped circular plate and accuracy of its approximate analytical solutions ${ }^{\dagger}$
}

\author{
Yin Zhang* \\ State Key Laboratory of Nonlinear Mechanics (LNM), Institute of Mechanics, Chinese Academy of Sciences, Beijing 100190, China
}

Received August 4, 2015; accepted September 9, 2015

\begin{abstract}
A different set of governing equations on the large deflection of plates are derived by the principle of virtual work (PVW), which also leads to a different set of boundary conditions. Boundary conditions play an important role in determining the computation accuracy of the large deflection of plates. Our boundary conditions are shown to be more appropriate by analyzing their difference with the previous ones. The accuracy of approximate analytical solutions is important to the bulge/blister tests and the application of various sensors with the plate structure. Different approximate analytical solutions are presented and their accuracies are evaluated by comparing them with the numerical results. The error sources are also analyzed. A new approximate analytical solution is proposed and shown to have a better approximation. The approximate analytical solution offers a much simpler and more direct framework to study the plate-membrane transition behavior of deflection as compared with the previous approaches of complex numerical integration.
\end{abstract}

approximate analytical solutions, large deflection, plate, membrane, bulge and blister tests

PACS number(s): 46.70.-p, 46.70.De, 62.20.F-, 68.55.-a, 85.85.+j

Citation: Y. Zhang, Large deflection of clamped circular plate and accuracy of its approximate analytical solutions, Sci. China-Phys. Mech. Astron. 59, 624602 (2016), doi: 10.1007/s11433-015-5751-y

\section{Introduction}

Bulge test [1-12] and blister test [13-17] are two reliable methods frequently used to extract thin film mechanical properties. Residual stress and its gradients are frequently encountered in the film/substrate composite structures fabricated by wafer-bonding, low-pressure chemical vapor deposition (LPCVD), etching, sputtering and epitaxial growth [1820]. When a film is separated from its substrate, the presence of residual stress and its gradients causes film to wrinkle or curl severely $[2,6]$. Because the uniaxial tensile testing of free-standing films characterizes the mechanical properties of those without residual stress and gradients [6], the presence of residual stress and gradients, which has significant impact on the film mechanical properties, makes it

*Corresponding author (email: zhangyin@ Inm.imech.ac.cn)

$\dagger$ Recommended by YaPu Zhao (Associate Editor) very difficult or impossible for the uniaxial tensile testing. Furthermore, for films made of compliant materials, the uniaxial tensile testing is often complicated by gripping difficulties, visco-elastic creep racheting, and other undesirable mechanical responses $[21,22]$. By etching a substrate $[6,9]$ or depositing/clamping a film on an orifice $[1,2,17]$, a freestanding structure is formed and transverse load can be applied via an indenter $[6,22,23]$ or pressure $[1,2,17]$. In the bulge and blister tests, by measuring the film deflection together with the load, the mechanical properties such as Young's modulus, Poisson's ratio and residual stress can be found. Because the film suspended length/radius is allowed to vary with the load in a blister test, other properties such as the film/substrate adhesion energy can also be measured [17, 23].

In the bulge and blister tests, film often experiences large deflection. Because the film thickness is very small compared 
with its other dimensions, the large deflection is usually geometrically nonlinear and elastic. The film large deflection is governed by the von Kármán equations [24], which also applies to a pressure sensor [9,25], a micro-valve [26], a microelectromechanical systems (MEMS) device [27] and the thin film delamination induced by buckling [28, 29]. Recently, the von Kármán equations are used to study the deformation of a graphene bubble [30]. Bubbles are frequently found at the graphene/silicon oxide interface [31]. Strain engineering is an effective method to alter the electronic properties of graphene, which, in this case, is to manipulate the deformation of a graphene bubble. Because of deformation, a quasiconstant pseudo-magnetic field can be generated to open a sizable gap on the electronic spectrum [31] and can thus significantly enhance the graphene application in digital electronics. A circular graphene bubble can also be used in optics as an adaptive-focus lens by controlling its deformation [31]. The nonlinear von Kármán equations with the high-order derivatives, according to Boudaoud et al. [32], are "notoriously difficult", mainly due to the fact that there are two types of deformations: bending and stretching associated with the energies that can vary with the difference of orders of magnitude. The demand for the approximate analytical solutions is huge because of its wide application. The continuous efforts of deriving those solutions for more than one hundred years are shown in Tables 1 and 2 [33-43]. However, as seen in Tables 1 and 2, different researchers obtained different approximate analytical solutions. The accuracy of these solu- tions is of great importance for the bulge and blister tests to extract the mechanical properties of a material. Sheplak and Dugundji [44] found that with the increase of initial tension, the plate deflection experiences a transition from the plate behavior (bending-dominant) to the membrane (stretchingdominant) behavior and proposed a dimensionless parameter to indicate the transition. Komaragiri et al. [21] further differentiated the membrane behavior as two parts: linear and nonlinear membrane behaviors and used two dimensionless parameters to indicate the transition. The transition is often called linear-cubic transition [14], which mathematically indicates the transition from $w_{o} \propto q$ to $w_{o}^{3} \propto q[21,44]\left(w_{o}\right.$ is the plate center displacement and $q$ is the transverse loading pressure). As seen in Tables 1 and 2, most researchers derived the same approximate solution for the linear range but none is the same for the cubic range. The approximate analytical solutions start with assuming certain deflection shape and radial displacement shape of a plate, which often violates the boundary conditions of von Kármán equations and thus causes the error [14].

In this study, a new set of boundary conditions are derived by the principle of virtual work (PVW), which shows some difference with the previous ones. An analysis is also presented to show that the new boundary conditions are more appropriate. Besides the violation of boundary conditions, those assumed shapes also violate the governing equations, which is another error source. An approximate analytical solution is presented by making the radial displacement shape

Table 1 Plate model: Different approximate analytical solutions of $q=K_{1} w_{o}+K_{2}\left(\epsilon_{o}\right) w_{o}+K_{3} w_{o}^{3}$

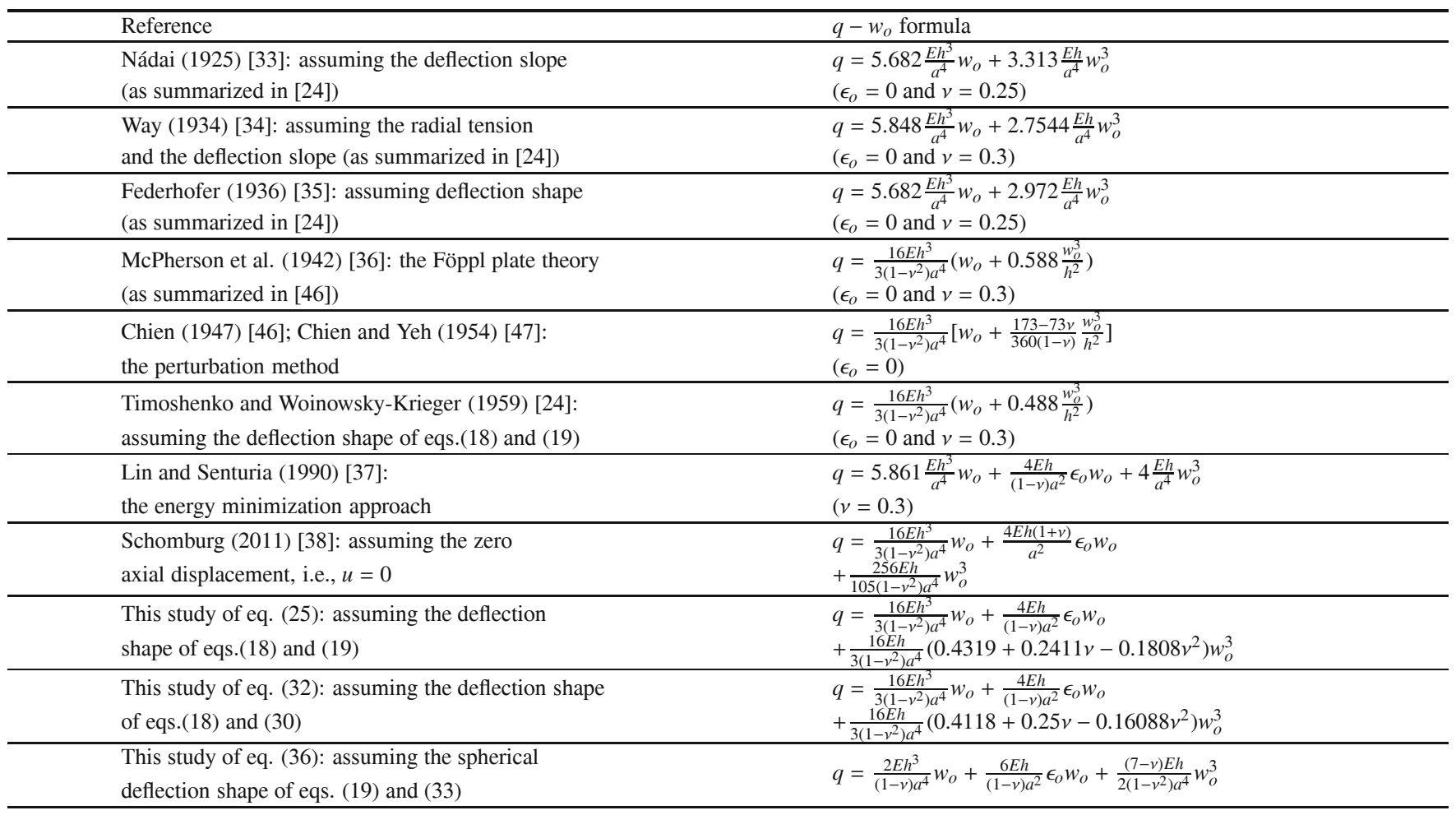


Table 2 Membrane model: Different approximate analytical solutions of $q=K_{2}\left(\epsilon_{o}\right) w_{o}+K_{3} w_{o}^{3}$

\begin{tabular}{|c|c|}
\hline Reference & $q-w_{o}$ formula \\
\hline Hencky (1915) [39]; Gent and Lewandowsky (1987) [40]: & $q=\frac{E h}{\left(\sum_{n=0}^{\infty} A_{2 n}\right)^{3} a^{4}} w_{o}^{3} \approx 3.447 \frac{E h}{a^{4}} w_{o}^{3}$ a) \\
\hline assuming the spherical shape of eq. (33) & $\left(\epsilon_{o}=0\right.$ and $\left.v=0.3\right)$ \\
\hline Beams (1959) [41]: the free-body diagram analysis & $q=\frac{4 E h}{(1-v) a^{2}} \epsilon_{o} w_{o}+3.56 \frac{E h}{a^{4}} w_{o}^{3}$ \\
\hline (as summarized in [13] ) & $(v=0.25)$ \\
\hline Allen and Senturia (1988) [42]: the fracture mechanics approach & $q=\frac{4 E h}{(1-v) a^{2}} \epsilon_{o} w_{o}+3.81 \frac{E h}{a^{4}} w_{o}^{3}(v=0.3)$ \\
\hline Small and Nix (1992) [1]: assuming the spherical shape of eq. (33) & $q=\frac{(7-v) E h}{3(1-v) a^{4}} w_{o}^{3}\left(\epsilon_{o}=0\right)$ \\
\hline Small and Nix (1992) [1]: free-body diagram analysis & $q=\frac{4 E h}{(1-v) a^{2}} \epsilon_{o} w_{o}+\frac{8 E h}{3(1-v) a^{4}} w_{o}^{3}$ \\
\hline Sizemore et al. (1995) [43]: the fracture mechanics approach & $q=\frac{4 E h}{(1-v) a^{2}} \epsilon_{o} w_{o}+3.59 \frac{E h}{a^{4}} w_{o}^{3}(v=0.3)$ \\
\hline Williams (1997) [13]: the fracture mechanics approach & $q=\frac{4 E h}{(1-\gamma) a^{2}} \epsilon_{o} w_{o}+\frac{2 E h}{(1-\gamma) a^{4}} w_{o}^{3}$ \\
\hline $\begin{array}{l}\text { Wan et al. (2003) [14]: assuming constant } \\
\text { radial and tangential stresses inside film }\end{array}$ & $q=\frac{4 E h}{\left(1-v^{2}\right) a^{2}} \epsilon_{o} w_{o}+\frac{2 E h}{\left(1-v^{2}\right) a^{4}} w_{o}^{3}$ \\
\hline Hsu et al. (2010) [11]: the energy minimization approach & $q=\frac{4 E h}{(1-v) a^{2}} \epsilon_{o} w_{o}+\frac{(7-v) E h}{3(1-v) a^{4}} w_{o}^{3}$ \\
\hline
\end{tabular}

a) where $A_{0}=1 / B_{0}, A_{2}=1 /\left(2 B_{0}^{4}\right), A_{4}=5 /\left(9 B_{0}^{7}\right), A_{6}=55 /\left(72 B_{0}^{10}\right), A_{8}=7 /\left(6 B_{0}^{13}\right)$ and so on with $B_{0}=1.713$ for $v=0.3$.

to exactly satisfy the governing equation with a given deflection shape. This new approximate analytical solution is shown to achieve a better approximation. As noticed by Small and Nix [1], most of previous studies on those approximate analytical solutions assume the Poisson's ratio as a constant of $v=0.25$ or $v=0.3$, which can significantly simplify the derivation. However, the generality is lost. Therefore, we derive the approximate analytical solutions by treating the Poisson's ratio as a variable rather than a constant. With the framework of the approximate analytical solution, the platemembrane transition behavior can be analyzed and grasped much more easily. Two new dimensionless parameters are also proposed to characterize the transition.

\section{Governing equations}

Figure 1(a) shows a circular plate of radius $a$ and thickness $h$, under an initial in-plane tension $N_{o}$ and a uniform transverse pressure $q$. The radial and tangential midplane strains are given as follows by using the kinematic assumptions of von Kármán plate theory [24]:

$$
\begin{aligned}
& \epsilon_{r}=\frac{N_{r}-v N_{t}}{E h}=\frac{\mathrm{d} u}{\mathrm{~d} r}+\frac{1}{2}\left(\frac{\mathrm{d} w}{\mathrm{~d} r}\right)^{2}+\epsilon_{o}, \\
& \epsilon_{t}=\frac{N_{t}-v N_{r}}{E h}=\frac{u}{r}+\epsilon_{o},
\end{aligned}
$$

where $N_{r}$ and $N_{t}$ are the radial and tangential tensions, respectively. $N_{r}$ and $N_{t}$ can be decomposed as $N_{r}=N_{o}+S_{r}$ and $N_{t}=N_{o}+S_{t}$ [44], where $S_{r}$ and $S_{t}$ are the incremental changes from $N_{o} . E$ and $v$ are the Young's modulus and Poisson's ratio of the plate, respectively. $u$ and $w$ are the radial and transverse displacements of plate, respectively. $\epsilon_{o}$ is the initial strain due to $N_{o}$, which has the following relation:

$$
\epsilon_{o}=\frac{N_{o}}{Y h}=\frac{(1-v) N_{o}}{E h}
$$

where $Y=E /(1-v)$ is the biaxial modulus. The plate bending energy of $V_{b}$ is given as follows [24]:

$$
\begin{aligned}
V_{b} & =\frac{D}{2} \int_{0}^{2 \pi} \int_{0}^{a}\left[\left(\frac{\mathrm{d}^{2} w}{\mathrm{~d} r^{2}}\right)^{2}+\frac{1}{r^{2}}\left(\frac{\mathrm{d} w}{\mathrm{~d} r}\right)^{2}+\frac{2 v}{r} \frac{\mathrm{d} w}{\mathrm{~d} r} \frac{\mathrm{d}^{2} w}{\mathrm{~d} r^{2}}\right] r \mathrm{~d} r \mathrm{~d} \theta \\
& =\pi D \int_{0}^{a}\left[\left(\frac{\mathrm{d}^{2} w}{\mathrm{~d} r^{2}}\right)^{2}+\frac{1}{r^{2}}\left(\frac{\mathrm{d} w}{\mathrm{~d} r}\right)^{2}+\frac{2 v}{r} \frac{\mathrm{d} w}{\mathrm{~d} r} \frac{\mathrm{d}^{2} w}{\mathrm{~d} r^{2}}\right] r \mathrm{~d} r
\end{aligned}
$$

where $D=E h^{3} / 12\left(1-v^{2}\right)$ is the plate bending stiffness. Here $w$ is assumed axisymmetric, i. e., $w=w(r)$. The plate stretching energy of $V_{s}$ is given as follows:

$$
\begin{aligned}
V_{s} & =2 \pi \int_{0}^{a}\left(\frac{N_{r} \epsilon_{r}}{2}+\frac{N_{t} \epsilon_{t}}{2}\right) r \mathrm{~d} r \\
& =\frac{\pi E h}{1-v^{2}} \int_{0}^{a}\left(\epsilon_{r}^{2}+\epsilon_{t}^{2}+2 v \epsilon_{r} \epsilon_{t}\right) r \mathrm{~d} r .
\end{aligned}
$$
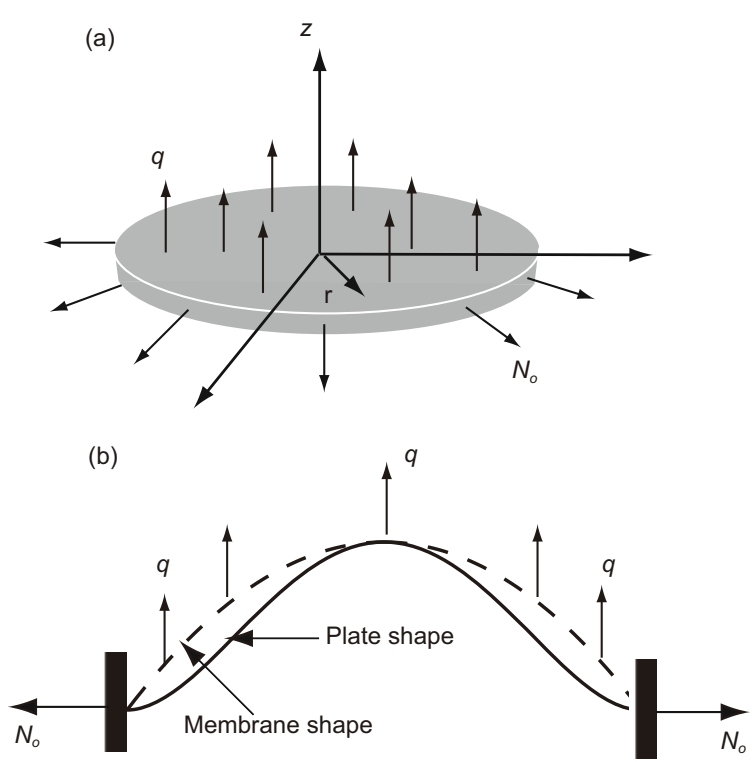

Figure 1 (a) The coordinate system and schematic diagram of a circular plate with initial in-plane tension of $N_{o}$ and transverse pressure of $q$. (b) The plate and membrane deflection shapes. 
The substitution of $\epsilon_{r}$ and $\epsilon_{t}$ of eq. (1) into the above equation is quite lengthy and is omitted here. $W_{e}$ is the external work done by $q$ and $N_{o}$, which is given as follows:

$$
W_{e}=2 \pi q \int_{0}^{a} w r \mathrm{~d} r+2 \pi a \int_{0}^{a} N_{o} \delta_{D}(r-a) u \mathrm{~d} r
$$

where $\delta_{D}(r-a)$ is the Dirac delta function. By applying the principle of virtual work (PVW), i.e., $\delta\left(V_{b}+V_{s}-W_{e}\right)=0$, the following governing equations and boundary conditions are derived:

$$
\begin{aligned}
& \frac{\mathrm{d}^{4} w}{\mathrm{~d} r^{4}}+\frac{2}{r} \frac{\mathrm{d}^{3} w}{\mathrm{~d} r^{3}}-\frac{1}{r^{2}} \frac{\mathrm{d}^{2} w}{\mathrm{~d} r^{2}}+\frac{1}{r^{3}} \frac{\mathrm{d} w}{\mathrm{~d} r} \\
= & \frac{N_{o}}{D}\left(\frac{1}{r} \frac{\mathrm{d} w}{\mathrm{~d} r}+\frac{\mathrm{d}^{2} w}{\mathrm{~d} r^{2}}\right)+\frac{12}{h^{2}}\left[\frac{1}{r} \frac{\mathrm{d} u}{\mathrm{~d} r} \frac{\mathrm{d} w}{\mathrm{~d} r}+\frac{\mathrm{d}^{2} u}{\mathrm{~d} r^{2}} \frac{\mathrm{d} w}{\mathrm{~d} r}\right. \\
& +\frac{\mathrm{d} u}{\mathrm{~d} r} \frac{\mathrm{d}^{2} w}{\mathrm{~d} r^{2}}+\frac{1}{2 r}\left(\frac{\mathrm{d} w}{\mathrm{~d} r}\right)^{3}+\frac{3}{2}\left(\frac{\mathrm{d} w}{\mathrm{~d} r}\right)^{2} \frac{\mathrm{d}^{2} w}{\mathrm{~d} r^{2}} \\
& \left.+\frac{v}{r}\left(\frac{\mathrm{d} u}{\mathrm{~d} r} \frac{\mathrm{d} w}{\mathrm{~d} r}+u \frac{\mathrm{d}^{2} w}{\mathrm{~d} r^{2}}\right)\right]+\frac{q}{D},
\end{aligned}
$$

and

$$
\frac{\mathrm{d}^{2} u}{\mathrm{~d} r^{2}}+\frac{1}{r} \frac{\mathrm{d} u}{\mathrm{~d} r}-\frac{u}{r^{2}}=-\frac{1-v}{2 r}\left(\frac{\mathrm{d} w}{\mathrm{~d} r}\right)^{2}-\frac{\mathrm{d} w}{\mathrm{~d} r} \frac{\mathrm{d}^{2} w}{\mathrm{~d} r^{2}}
$$

The six boundary conditions are derived as follows:

$$
\begin{aligned}
& u(0)=0, \quad \frac{\mathrm{d} w}{\mathrm{~d} r}(0)=0, \quad w(a)=0, \\
& \frac{\mathrm{d} w}{\mathrm{~d} r}(a)=0, \quad u(a)=0, \\
& \left.\frac{E h}{1-v^{2}}\left[\frac{\mathrm{d} u}{\mathrm{~d} r}+\frac{1}{2}\left(\frac{\mathrm{d} w}{\mathrm{~d} r}\right)^{2}+\frac{v}{r} u\right]\right|_{r=a}=0 .
\end{aligned}
$$

It is noticed that $N_{o}$ has no impact on eq. (7). Here eq. (6) is a fourth order differential equation. The corresponding second and third order forms of eq. (6) are derived here for a comparison. The balance of force gives the following equation [24]:

$$
Q_{r}=-N_{r} \frac{\mathrm{d} w}{\mathrm{~d} r}-\frac{q r}{2}
$$

where $Q_{r}$ is the shearing force given as follows:

$$
Q_{r}=-D\left(\frac{\mathrm{d}^{3} w}{\mathrm{~d} r^{3}}+\frac{1}{r} \frac{\mathrm{d}^{2} w}{\mathrm{~d} r^{2}}-\frac{1}{r^{2}} \frac{\mathrm{d} w}{\mathrm{~d} r}\right)
$$

$N_{r}$ is derived from eq. (1) as follows:

$$
\begin{aligned}
N_{r} & =\frac{E h}{1-v^{2}}\left(\epsilon_{r}+v \epsilon_{t}\right)=S_{r}+N_{o} \\
& =\frac{E h}{1-v^{2}}\left[\frac{\mathrm{d} u}{\mathrm{~d} r}+\frac{1}{2}\left(\frac{\mathrm{d} w}{\mathrm{~d} r}\right)^{2}+v \frac{u}{r}\right]+N_{o} .
\end{aligned}
$$

With the substitution of eqs. (10) and (11) into eq. (9), the third order governing equation is derived as follows:

$$
\frac{\mathrm{d}^{3} w}{\mathrm{~d} r^{3}}+\frac{1}{r} \frac{\mathrm{d}^{2} w}{\mathrm{~d} r^{2}}-\frac{1}{r^{2}} \frac{\mathrm{d} w}{\mathrm{~d} r}
$$

$$
=\frac{12}{h^{2}}\left[\frac{\mathrm{d} u}{\mathrm{~d} r}+\frac{1}{2}\left(\frac{d w}{\mathrm{~d} r}\right)^{2}+v \frac{u}{r}\right] \frac{\mathrm{d} w}{\mathrm{~d} r}+\frac{N_{o}}{D} \frac{\mathrm{d} w}{\mathrm{~d} r}+\frac{q r}{2 D} .
$$

It is readily found that the fourth order governing equation of eq. (6) can be obtained by doing the following operation of $\mathrm{d} / \mathrm{d} r\{$ eq. (12) $\}+1 / \mathrm{r}$ \{eq. (12) \}. Eqs. (12) and (7) are the set of governing equations obtained by Timoshenko and Woinowsky-Krieger [24]. The third order governing equation of eq. (12) can be further reduced to the following second order equation by the simple substitution of $\theta=\mathrm{d} w / \mathrm{d} r$ :

$$
\frac{\mathrm{d}^{2} \theta}{\mathrm{d} r^{2}}+\frac{1}{r} \frac{\mathrm{d} \theta}{\mathrm{d} r}-\frac{1}{r^{2}} \theta=\frac{S_{r}+N_{o}}{D} \theta+\frac{q r}{2 D} .
$$

Here $S_{r} / D=\frac{12}{h^{2}}\left[\frac{\mathrm{d} u}{\mathrm{~d} r}+\frac{1}{2}\left(\frac{\mathrm{d} w}{\mathrm{~d} r}\right)^{2}+v \frac{u}{r}\right]$ has been used. If $S_{r}$ and $S_{t}$ are used as unknown variables, eq. (7) can be decomposed into the following two first order differential equations

$$
\frac{\mathrm{d} S_{r}}{\mathrm{~d} r}+\frac{S_{r}-S_{t}}{r}=0
$$

and

$$
\frac{\mathrm{d} S_{t}}{\mathrm{~d} r}-\frac{S_{r}-S_{t}}{r}+\frac{E h}{2 r}\left(\frac{\mathrm{d} w}{\mathrm{~d} r}\right)^{2}=0 .
$$

Physically, eq. (14) indicates the plate equilibrium of radial direction and eq. (15) is just the compatibility condition [26]. Eqs. (13), (14) and (15) are the three governing equations adopted in references $[26,44]$. It is noticed that if the nonlinear term of $S_{r} \theta / D$ is ignored, eq. (13) becomes the following by multiplying $r^{2}$ :

$$
r^{2} \frac{\mathrm{d}^{2} \theta}{\mathrm{d} r^{2}}+r \frac{\mathrm{d} \theta}{\mathrm{d} r}-\left(1+N_{o} r^{2}\right) \theta=\frac{q r^{3}}{2 D} .
$$

Here eq. (16) is a modified Bessel equation with an analytical solution [44].

The four boundary conditions for these one second order and two first order equations are given by Sheplak and Dugundji [44] as follows:

$$
\theta(0)=0, \quad S_{r}(0)=S_{t}(0) ; \quad \theta(a)=0, \quad u(a)=0 .
$$

Here we need to have a discussion on an issue raised by eq. (17), which gives the boundary condition of $S_{r}(0)=S_{t}(0)$. By definition, $S_{r}=\frac{E h}{1-v^{2}}\left(\epsilon_{r}+v \epsilon_{t}\right)=\frac{E h}{1-v^{2}}\left[\frac{\mathrm{d} u}{\mathrm{~d} r}+\frac{1}{2}\left(\frac{\mathrm{d} w}{\mathrm{~d} r}\right)^{2}+v \frac{u}{r}\right]$ and $S_{t}=\frac{E h}{1-v^{2}}\left(\epsilon_{t}+v \epsilon_{r}\right)=\frac{E h}{1-v^{2}}\left[\frac{u}{r}+v \frac{\mathrm{d} u}{\mathrm{~d} r}+\frac{v}{2}\left(\frac{\mathrm{d} w}{\mathrm{~d} r}\right)^{2}\right]$. Because $\frac{\mathrm{d} w}{\mathrm{~d} r}(0)=0$ and $\lim _{r \rightarrow 0} \frac{u}{r}=\frac{\mathrm{d} u}{\mathrm{~d} r}$ due to L'Hospital rule, $\lim _{r \rightarrow 0} S_{r}=\lim _{r \rightarrow 0} S_{t}=\frac{E h}{1-\gamma} \frac{\mathrm{d} u}{\mathrm{~d} r}$. Therefore, $S_{r}(0)=S_{t}(0)$ is a natural result rather than a boundary condition needed to be enforced. Physically, it is not that hard to think that at the plate center of $r=0$, there is no difference between the tangential and radial tensions, i.e., $S_{r}(0)=S_{t}(0)$. On the other hand, $u(0)=0$ as given in eq. (8) must be enforced as a boundary condition [45]. Otherwise, $\epsilon_{t}=u / r+\epsilon_{o}$ together with $S_{r}$ and $S_{t}$ will become unphysically infinite at $r=0$. Chien [46], Chien and Yeh [47] also used $S_{r}$ and $S_{t}$ as the unknown variables; $S_{r}(0)$ is a finite quantity and one of their enforced boundary conditions. 


\section{Approximate analytical solutions}

Timoshenko and Woinowsky-Krieger assumed the following shapes for $w$ and $u$ [24]

$$
w=w_{o}\left(1-\frac{r^{2}}{a^{2}}\right)^{2},
$$

and

$$
u=r(a-r)\left(C_{1}+C_{2} r\right),
$$

where $w_{o}=w(0)$ is the deflection of the plate center; $C_{1}$ and $C_{2}$ are the unknown constants to be determined. By substituting eqs. (18) and (19) into eqs. (3) and (4), we have

$$
\begin{gathered}
V_{b}=\frac{32}{3} \pi D \frac{w_{o}^{2}}{a^{2}} \\
V_{s}=\frac{\pi E h}{1-v^{2}}\left[\frac{a^{4} C_{1}^{2}}{4}+\frac{3 a^{5} C_{1} C_{2}}{10}\right. \\
+\frac{7 a^{6} C_{2}^{2}}{60}+w_{o}^{2} a(-0.146+0.2603 v) C_{1} \\
+w_{o}^{2} a^{2}(0.012+0.13968 v) C_{2} \\
\left.+\frac{32}{105} \frac{w_{o}^{4}}{a^{2}}+\frac{2(1+v)}{3} \epsilon_{o} w_{o}^{2}+\epsilon_{o}^{2} a^{2}\right]
\end{gathered}
$$

For equilibrium, the total strain energy $V_{t}=V_{b}+V_{s}$ needs to be a minimum, which leads to the following [24]:

$$
\frac{\partial V_{t}}{\partial C_{1}}=\frac{\partial V_{s}}{\partial C_{1}}=0, \quad \frac{\partial V_{t}}{\partial C_{2}}=\frac{\partial V_{s}}{\partial C_{2}}=0,
$$

from which $C_{1}$ and $C_{2}$ are found as:

$$
\begin{aligned}
C_{1}= & {\left[\frac{35}{4}(0.146-0.2603 v)\right.} \\
& \left.+\frac{45}{4}(0.0127+0.13968 v)\right] \frac{w_{o}^{2}}{a^{3}}, \\
C_{2}= & {\left[-\frac{45}{4}(0.146-0.2603 v)\right.} \\
& \left.-\frac{75}{4}(0.0127+0.13968 v)\right] \frac{w_{o}^{2}}{a^{4}} .
\end{aligned}
$$

Now substitute eq. (23) into eqs. (21) and (22), and apply the principle of virtual displacement (PVD) [24], i.e.,

$$
\frac{\mathrm{d} V_{t}}{\mathrm{~d} w_{o}} \delta w_{o}=2 \pi \int_{0}^{a} q \delta w r \mathrm{~d} r=2 \pi q \delta w_{o} \int_{0}^{a}\left(1-\frac{r^{2}}{a^{2}}\right)^{2} r \mathrm{~d} r
$$

which leads to the following:

$$
\begin{aligned}
& {\left[1+\frac{3}{4}\left(\frac{a}{h}\right)^{2}(1+v) \epsilon_{o}\right] w_{o}+\left(0.4319+0.2411 v-0.1808 v^{2}\right) \frac{w_{o}^{3}}{h^{2}}} \\
& =\frac{q a^{4}}{64 D} .
\end{aligned}
$$

The first term $w_{o}$ is due to bending; the second term $\frac{3}{4}\left(\frac{a}{h}\right)^{2}(1+$ $v) \epsilon_{o} w_{o}$ is due to the initial midplane stretching; the third term $\left(0.4319+0.2411 v-0.1808 v^{2}\right) \frac{w_{o}^{3}}{h^{2}}$ is due to the further midplane stretching from the initial stretching. Eq. (25) is a cubic equation with the analytical Cardan solution [48]. When $\epsilon_{o}=0$ and $v=0.3$, the above equation recovers the following one obtained by Timoshenko and Woinowsky-Krieger [24]:

$$
w_{o}+0.488 \frac{w_{o}^{3}}{h^{2}}=\frac{q a^{4}}{64 D} .
$$

Although Timoshenko and Woinowsky-Krieger [24] did not include the initial midplane stretching term, we refer to eq. (25) as the Timoshenko solution, which together with other approximate solutions is the cornerstone for the bulge test to extract the material properties [1].

The assumed deflection shape of eq. (18) satisfies the clamped boundary conditions at $r=a$, which are $w(a)=$ $\mathrm{d} w / \mathrm{d} r(a)=0$; at the plate center of $r=0$, eq. (18) also satisfies $\mathrm{d} w / \mathrm{d} r(0)=0$. Eq. (18) is thus referred to as the plate shape and shown in Figure 1(b). The $u$ solution form of eq. (19) satisfies the boundary conditions of $u(0)=0$ and $u(a)=0$. There are two major mechanisms causing the Timoshenko approximation to deviate (slightly) from the numerical solution. One is the truncation errors caused by the transverse and radial displacement assumptions of eqs. (18) and (19). To reduce/eliminate the error, Way $[24,34]$ expanded $S_{r}$ and $\mathrm{d} w / \mathrm{d} r$ into the power series, whose convergence was proved by Zheng and Zhou [49]. Similarly, Timoshenko and Woinowsky-Krieger provided the following general form for $u$ [24]:

$$
u=r(a-r)\left(C_{1}+C_{2} r+C_{3} r^{2}+\cdots\right) .
$$

However, it is extremely difficult if not impossible to use the solution form of eq. (27) to derive something like eq. (25). The other mechanism is that the solution form of eq. (19) actually does not satisfy the governing equation of eq. (7). Here we propose a new approximate form of $u$ to improve the accuracy. The deflection shape of $w$ is still approximated by eq. (18) and substituted into eq. (7), which gives the following equation:

$$
\begin{aligned}
\frac{\mathrm{d}^{2} u}{\mathrm{~d} r^{2}}+\frac{1}{r} \frac{\mathrm{d} u}{\mathrm{~d} r}-\frac{u}{r^{2}}= & \frac{(-24+8 v) w_{o}^{2}}{a^{4}} r+\frac{(80-16 v) w_{o}^{2}}{a^{6}} r^{3} \\
& +\frac{(-56+8 v) w_{o}^{2}}{a^{8}} r^{5} .
\end{aligned}
$$

$u$ can now be solved as follows:

$$
\begin{aligned}
u= & C_{1}+C_{2} r+(-3+v) \frac{w_{o}^{2}}{a^{4}} r^{3}+\frac{(10-2 v) w_{o}^{2}}{3 a^{6}} r^{5} \\
& +\frac{(-7+v) w_{o}^{2}}{6 a^{8}} r^{7} .
\end{aligned}
$$

Here $C_{1}+C_{2} r$ are the homogeneous solution and the last three terms of $(-3+v) w_{o}^{2} r^{3} / a^{4}+(10-2 v) w_{o}^{2} r^{5} /\left(3 a^{6}\right)+(-7+$ v) $w_{o}^{2} r^{7} /\left(6 a^{8}\right)$ are the particular solutions. $C_{1}$ and $C_{2}$ are the constants determined by the boundary conditions of $u(0)=0$ and $u(a)=0$, which leads to the following:

$$
u=\frac{(5-3 v) w_{o}^{2}}{6 a^{2}} r+(-3+v) \frac{w_{o}^{2}}{a^{4}} r^{3}+\frac{(10-2 v) w_{o}^{2}}{3 a^{6}} r^{5}
$$




$$
+\frac{(-7+v) w_{o}^{2}}{6 a^{8}} r^{7}
$$

Clearly, the $u$ solution form of eq. (30) is very different from Timoshenko's of eq. (19). Now substitute eqs. (18) and (30) into eqs. (3) and (4), $V_{b}$ keeps unchanged as given by eq. (21) and $V_{s}$ becomes the following:

$$
V_{s}=\frac{w_{o}^{4}}{a^{2}}\left(0.183+0.1111 v-0.0715 v^{2}\right)+\frac{2}{3}(1+v) \epsilon_{o} w_{o}^{2} .
$$

By applying PVD of eq. (24), the following equation is obtained:

$$
\begin{aligned}
& {\left[1+\frac{3}{4}\left(\frac{a}{h}\right)^{2}(1+v) \epsilon_{o}\right] w_{o}+\left(0.4118+0.25 v-0.16088 v^{2}\right) \frac{w_{o}^{3}}{h^{2}}} \\
& =\frac{q a^{4}}{64 D}
\end{aligned}
$$

It is noticed that the only difference between eqs. (25) and (32) is the cubic term. Compared with eq. (25), eq. (32) is more accurate as discussed later because its corresponding radial displacement of eq. (30) satisfies the governing equation of eq. (28). In contrast, the radial displacement form of eq. (19) does not.

Instead of assuming the plate deflection shape of eq. (18), Lin [5] presented the following deflection shape:

$$
w=w_{o}\left(1-\frac{r^{2}}{a^{2}}\right)
$$

which is a spherical membrane shape as plotted in Figure 1(b). Clearly, this assumed shape does not satisfy the clamped condition of zero slope. At the same time, the radial displacement still keeps the form of eq. (19). Because the change of the solution form of $w$, the bending energy of $V_{b}$ and the stretching energy of $V_{s}$ become the following:

$$
\begin{aligned}
V_{b}= & 4 \pi D(1+v) \frac{w_{o}^{2}}{a^{2}}, \\
V_{s}= & \frac{\pi E h}{1-v^{2}}\left[\frac{a^{4} C_{1}^{2}}{4}+\frac{3 a^{5} C_{1} C_{2}}{10}+\frac{7 a^{6} C_{2}^{2}}{60}\right. \\
& +\frac{v-3}{5} w_{o}^{2} a C_{1}+\frac{2 v-6}{15} w_{o}^{2} a^{2} C_{2} \\
& \left.+\frac{2}{3} \frac{w_{o}^{4}}{a^{2}}+(1+v) \epsilon_{o} w_{o}^{2}+\epsilon_{o}^{2} a^{2}+\frac{v}{2} \epsilon_{o} a^{2}\right] .
\end{aligned}
$$

Compared with eq. (20), there are some minor changes on $V_{s}$. However, $V_{b}$ becomes significantly smaller with the membrane shape assumption. Now repeat the same procedures of minimizing the total strain energy and PVD, $C_{1}$ and $C_{2}$ are solved as follows:

$$
C_{1}=a C_{2}=\frac{(3-v) w_{o}^{2}}{4 a^{3}}
$$

and the following cubic equation is obtained:

$$
\left[1+3\left(\frac{a}{h}\right)^{2}(1+v) \epsilon_{o}\right] w_{o}+\frac{7-v}{4} \frac{w_{o}^{3}}{h^{2}}=\frac{q a^{4}}{24 D(1+v)},
$$

which is referred to as Lin's approximation. Similarly, we can use the same approach of deriving eq. (32) to rederive the above equation by making the radial displacement to satisfy the governing equation with the assumed shape of eq. (33). However, it happens to be that this approach leads to the same equation as eq. (36). Using the same approximate shapes of eqs. (33) and (19), Small and Nix [1] derived the following equation, which is obtained by rearranging their eq. (11)

$$
\frac{7-v}{6} \frac{w_{o}^{3}}{h^{2}}=\frac{q a^{4}}{24 D(1+v)},
$$

which is referred to as the Small-Nix approximation. Compared with eq. (36), the linear terms are missing and the factor difference of the cubic term is also noticed. Small and Nix [1] also used the membrane model, in which there is no bending energy, to derive the following equation, which is obtained by rearranging their eq. (15)

$$
\frac{3}{4}\left(\frac{a}{h}\right)^{2}(1+v) \epsilon_{o} w_{o}+\frac{1}{2}(1+v) \frac{w_{o}^{3}}{h^{2}}=\frac{q a^{4}}{64 D},
$$

which is referred to as the membrane approximation. There is no real solution for $\epsilon_{o}<0$, i.e., the membrane structure cannot stand compression. However, it is not unusual at all that the residual stress inside a film is compressive [7] and the plate model has to be applied. Compared with the initial and further stretching terms presented in eqs. (25) and (32), there are some minor differences as $v$ changes from 0 to 0.5 . This fact indicates that the plate shape of eq. (18) is capable of capturing the membrane/stretching behavior accurately.

The approximate $q-w_{o}$ relation for a circular plate/membrane is often written as the following form of $q=\left(K_{1}+K_{2}\right) w_{o}+K_{3} w_{o}^{3}[1,3]$. Several of them are presented in Tables 1 and 2. Here $K_{1}, K_{2}$ and $K_{3}$ are associated with the three unknowns of $E, v$ and $\epsilon_{o}$. In a bulge test, $q$ and $w_{o}$ are measured together with the plate/membrane dimensions, only two parameters of $K_{1}+K_{2}$ and $K_{3}$ can be obtained by curve-fitting. This means that the unknown material properties of $E, v$ and $\epsilon_{o}$ in general cannot be extracted individually from the bulge test of a circular film [11]. However, Vlassak and Nix [3] showed that for a rectangular film, these three material parameters can be extracted by changing the film aspect ratio. Here the $K_{1} w_{o}$ term is associated with the plate bending. As seen from Table 1, Nadai and Federhofer [24] both got $K_{1}=5.682 E h^{3} / a^{4}$ for $v=0.25$; Way [24] got $K_{1}=5.848 E h^{3} / a^{4}$ for $v=0.3$. Our eqs. (25) and (32) find $K_{1}=16 E h^{3} /\left[3\left(1-v^{2}\right) a^{4}\right]$, which gives $K_{1}=5.689 E h^{3} / a^{4}$ for $v=0.25$ and $K_{1}=5.861 E h^{3} / a^{4}$ for $v=0.3$. It is an excellent agreement with Nadai, Federhofer and Way's. The $K_{2} w_{o}$ is associated with the residual strain $\epsilon_{o}$. As seen from Table 1, most studies including this one obtained $K_{2}=4 E h \epsilon_{o} /\left[(1-v) a^{2}\right]$. The $K_{3} w_{o}^{3}$ term is associated with further nonlinear stretching of mid-plane; the problem is on this $K_{3}$ value. As seen in Tables 1 and $2, K_{3}$ varies significantly. This is the general trend as observed by 
Wan et al. [14] that the approximate analytical solution fits the numerical/real solution quite well when the deflection is small, or say, $w_{o} \propto q$ and it deviates significantly when the deflection is large, i.e., $w_{o} \propto q^{1 / 3}$.

\section{Nondimensionalization}

To nondimensionalize, the following quantities are introduced [44]:

$$
\xi=\frac{r}{a}, \quad W=\frac{w}{h}, \quad W_{o}=\frac{w_{o}}{h}, \quad U=\frac{u}{h} .
$$

The governing equations of eqs. (6) and (7) are now nondimensionalized as follows:

$$
\begin{aligned}
& \frac{\mathrm{d}^{4} W}{\mathrm{~d} \xi^{4}}+\frac{2}{\xi} \frac{\mathrm{d}^{3} W}{\mathrm{~d} \xi^{3}}-\frac{1}{\xi^{2}} \frac{\mathrm{d}^{2} W}{\mathrm{~d} \xi^{2}}+\frac{1}{\xi^{3}} \frac{\mathrm{d} W}{\mathrm{~d} \xi} \\
= & \frac{N_{o} a^{2}}{D}\left(\frac{1}{\xi} \frac{\mathrm{d} W}{\mathrm{~d} \xi}+\frac{\mathrm{d}^{2} W}{\mathrm{~d} \xi^{2}}\right)+\frac{12 a}{h}\left[\frac{1}{\xi} \frac{\mathrm{d} U}{\mathrm{~d} \xi} \frac{\mathrm{d} W}{\mathrm{~d} \xi}\right. \\
& \left.+\frac{\mathrm{d}^{2} U}{\mathrm{~d} \xi^{2}} \frac{\mathrm{d} W}{\mathrm{~d} \xi}+\frac{\mathrm{d} U}{\mathrm{~d} \xi} \frac{\mathrm{d}^{2} W}{\mathrm{~d} \xi^{2}}+\frac{v}{\xi}\left(\frac{\mathrm{d} U}{\mathrm{~d} \xi} \frac{\mathrm{d} W}{\mathrm{~d} \xi}+U \frac{\mathrm{d}^{2} W}{\mathrm{~d} \xi^{2}}\right)\right] \\
+ & 12\left[\frac{1}{2 \xi}\left(\frac{\mathrm{d} W}{\mathrm{~d} \xi}\right)^{3}+\frac{3}{2}\left(\frac{\mathrm{d} W}{\mathrm{~d} \xi}\right)^{2} \frac{\mathrm{d}^{2} W}{\mathrm{~d} \xi^{2}}\right]+\frac{q a^{4}}{D h},
\end{aligned}
$$

and

$$
\frac{\mathrm{d}^{2} U}{\mathrm{~d} \xi^{2}}+\frac{1}{\xi} \frac{\mathrm{d} U}{\mathrm{~d} \xi}-\frac{U}{\xi^{2}}=-\frac{1-v}{2 \xi} \frac{h}{a}\left(\frac{\mathrm{d} W}{\mathrm{~d} \xi}\right)^{2}-\frac{h}{a} \frac{\mathrm{d} W}{\mathrm{~d} \xi} \frac{\mathrm{d}^{2} W}{\mathrm{~d} \xi^{2}} .
$$

The boundary conditions of eq. (8) now become the following dimensionless ones:

$$
\begin{aligned}
& U(0)=0, \quad \frac{\mathrm{d} W}{\mathrm{~d} \xi}(0)=0, \quad W(1)=0, \\
& \frac{\mathrm{d} W}{\mathrm{~d} \xi}(1)=0, \quad U(1)=0, \\
& {\left.\left[\frac{\mathrm{d} U}{\mathrm{~d} \xi}+\frac{1}{2} \frac{h}{a}\left(\frac{\mathrm{d} W}{\mathrm{~d} \xi}\right)^{2}+\frac{v}{\xi} U\right]\right|_{\xi=1}=0 .}
\end{aligned}
$$

eqs. (40) and (41) together with the boundary conditions of eq. (42) form a typical two-point boundary value problem, which can only be solved numerically $[21,44,50]$. Because of the terms associated with $\xi^{-1}, \xi^{-2}$ and $\xi^{-3}$, the numerical singularity, convergence problem and extreme sensitivity to the initial guess are encountered in the shooting method and the relaxation method is thus advised [21]. Sheplak and Dugundji [44] used the analytical solution of eq. (16) as their initial guess. Here our initial guess is based on the Timoshenko approximation of eq. (25) or the new approximation of eq. (32). It is worth pointing out these two approximations as the initial guess usually do not lead to the numerical solution within an acceptable error tolerance; our initial guess varies around these approximations; it is quite often that numerous trial-and-error tests are needed before a proper initial guess can be found.
The Timoshenko approximation of eq. (25) now becomes the following dimensionless one

$$
\begin{aligned}
& {\left[1+\frac{3}{4}\left(\frac{a}{h}\right)^{2}(1+v) \epsilon_{o}\right] W_{o}+\left(0.4319+0.2411 v-0.1808 v^{2}\right) W_{o}^{3}} \\
& =\frac{q a^{4}}{64 D h} .
\end{aligned}
$$

Once $W_{o}$ is solved from the above equation, the transverse and radial displacements are readily obtained from eqs. (18) and (19) in conjunction with eq. (23) as follows:

$$
\begin{aligned}
W= & W_{o}\left(1-\xi^{2}\right)^{2}, \\
U= & \left(\frac{h}{a}\right) W_{o}^{2}[(1.4204-0.7062 v) \xi \\
& +(-3.301+1.0156 v) \xi^{2} \\
& \left.-(-1.8806+0.3094 v) \xi^{3}\right] .
\end{aligned}
$$

The new approximate solution of eq. (32) presented in this study becomes the following dimensionless one:

$$
\begin{aligned}
& {\left[1+\frac{3}{4}\left(\frac{a}{h}\right)^{2}(1+v) \epsilon_{o}\right] W_{o}+\left(0.4118+0.25 v-0.16088 v^{2}\right) W_{o}^{3}} \\
& =\frac{q a^{4}}{64 D h}
\end{aligned}
$$

The transverse and radial displacements of eqs. (18) and (30) become the following:

$$
\begin{aligned}
W= & W_{o}\left(1-\xi^{2}\right)^{2}, \\
U= & \left(\frac{h}{a}\right) W_{o}^{2}\left[\frac{5-3 v}{6} \xi+(-3+v) \xi^{3}\right. \\
& \left.+\frac{10-2 v}{3} \xi^{5}+\frac{(-7+v)}{6} \xi^{7}\right] .
\end{aligned}
$$

Lin's approximation of eq. (36) becomes

$$
\left[1+3\left(\frac{a}{h}\right)^{2}(1+v) \epsilon_{o}\right] W_{o}+\frac{7-v}{4} W_{o}^{3}=\frac{q a^{4}}{24 D h(1+v)} .
$$

The transverse and radial displacements of eqs. (33) and (30) become the following in conjunction with eq. (35):

$$
W=W_{o}\left(1-\xi^{2}\right), \quad U=\frac{(-3+v) h}{4 a} W_{o}^{2} \xi\left(\xi^{2}-1\right) .
$$

The Small-Nix approximation of eq. (37) becomes the following dimensionless one:

$$
\frac{7-v}{6} W_{o}^{3}=\frac{q a^{4}}{24 D h(1+v)} .
$$

The transverse and radial displacements of the Small-Nix approximation share the same form of eq. (48) with Lin's; the difference is in $W_{o}$, which is solved by eqs. (47) and (49), respectively.

The membrane approximation of eq. (38) becomes the following dimensionless one:

$$
\frac{3}{4}\left(\frac{a}{h}\right)^{2}(1+v) \epsilon_{o} W_{o}+\frac{1}{2}(1+v) W_{o}^{3}=\frac{q a^{4}}{64 D h} .
$$




\section{Results and discussion}

In this study, $a / h$ is fixed as 20 and Poisson's ratio is taken as $v=0.3$. In Figures $2-4, \epsilon_{o}=1 \times 10^{-4}$ and $q / E=5 \times 10^{-5}$. The positive value of $\epsilon_{o}$ indicates the strain is tensile. In a bulge test, the initial compressive strain can cause the film wrinkling, which makes the property extraction of film very difficult $[1,2]$. Furthermore, in the modeling aspect, the membrane model can not take compression. Figure 2 plots the transverse displacements solved numerically and calculated by different approximations. The Timoshenko approximation and the new approximation of eq. (45) (almost) overlap each other; they are also the closest ones to the numerical solution. The Lin, membrane and Small-Nix approximations all overestimate the transverse displacement, or in other words, these three approximations are with the larger flexurality. As mentioned above and indicated in eq. (43) or (45), the plate stiffness consists of three parts: bending, initial stretching and further stretching. The bending energy difference can be seen in eqs. (21) and (34), which are calculated by different deflection forms of eqs. (18) and (33). Figure 3 plots the deflection slope of $W^{\prime}=\mathrm{d} W / \mathrm{d} \xi$. Clearly, only the Timoshenko approximation and the new approximation of eq. (45) satisfy the clamped condition of zero slope at $\xi=1$ and the other three violate. The membrane shape of eq. (33) violates the clamped condition of zero slope, which makes the structure more flexural than it should be. This is the mechanism responsible for the larger deflection of the Lin's approximation. The membrane approximation does not include the bending stiffness at all and thus makes the structure more flexural than Lin's approximation. The effect of the initial tensile stretching $\left(\epsilon_{o}\right)$, which stiffens the structure, is missing in the Small-Nix approximation and makes it the most flexural. Figure 4 plots the radial displacement $U$. It is noticed that $U$ obtained by the Lin and Small-Nix approximation is always positive. In contrast, the numerical study, Tim-

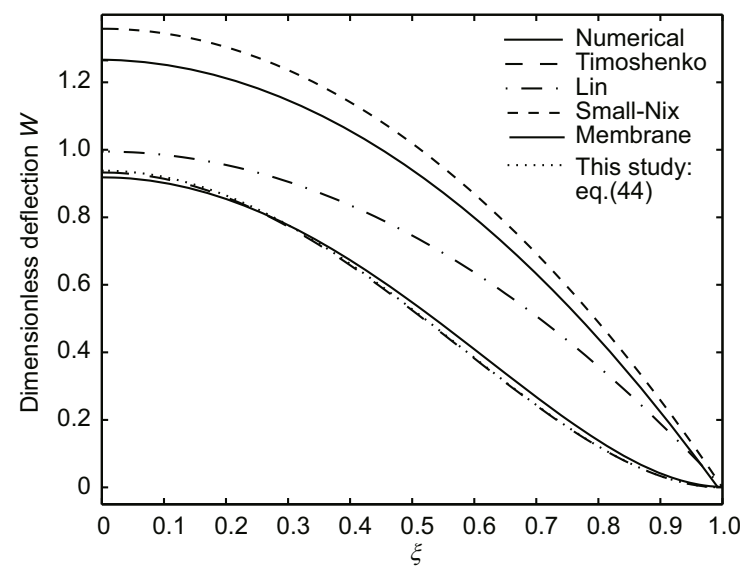

Figure 2 The plate deflections calculated by different approximate analytical solutions and numerical one when $\epsilon_{o}=10^{-4}, q / E=5 \times 10^{-5}$ and $a / h=20$.

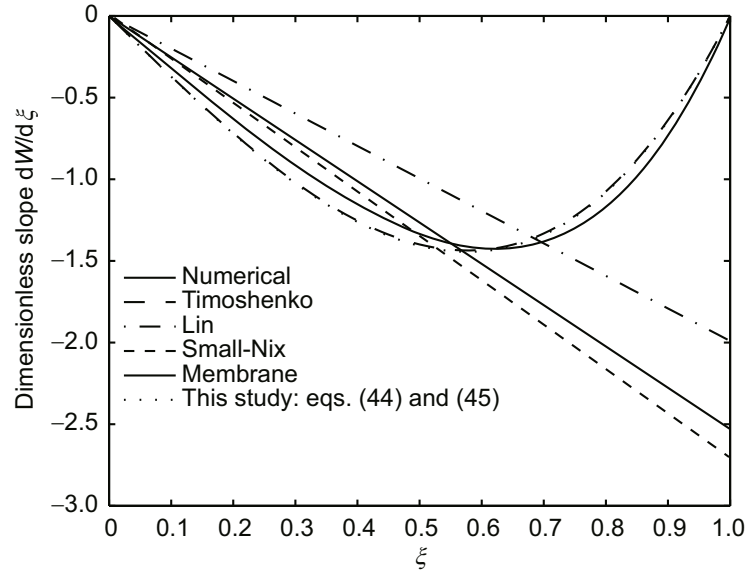

Figure 3 The slopes calculated by different approximate analytical solutions and numerical one when $\epsilon_{o}=10^{-4}, q / E=5 \times 10^{-5}$ and $a / h=20$.

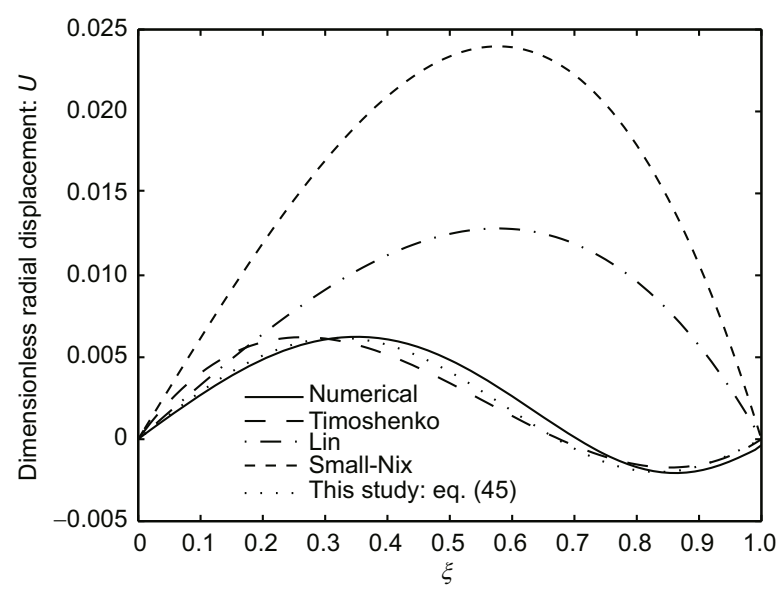

Figure 4 The plate in-plane radial displacements calculated by different approximate analytical solutions and numerical one when $\epsilon_{O}=10^{-4}$, $q / E=5 \times 10^{-5}$ and $a / h=20$.

oshenko approximation and the approximation of this study all indicate that $U$ is positive around $0 \leqslant \xi \leqslant 0.69$ and $U$ is negative around $0.69 \leqslant \xi \leqslant 1$, which has also been observed by Sheplak and Dugundji [44]. In that sense, the membrane shape approximation of eq. (33) fails to capture the variation of the radial displacement. It is also noticed that in Figure 4, $U$ given by our new approximation is closer to the numerical solution than Timoshenko's. Again, the explanation is that our radial displacement form of eq. (30) satisfies the governing equation of eq. (28) and Timoshenko's eq. (19) does not.

In Figures 5-7, larger $\epsilon_{o}=1.5 \times 10^{-3}$ and larger $q / E=$ $1 \times 10^{-4}$ are set. Figure 5 plots the transverse displacement and again, the Timoshenko and our approximations (almost) overlap; with the increase of initial strain and transverse pressure, these two approximations are still the closest ones to the numerical solution, then it is Lin's, membrane and Small-Nix approximations. The reason is still the same one as given above. Figure 6 presents the slopes. Once again, Lin's, mem- 


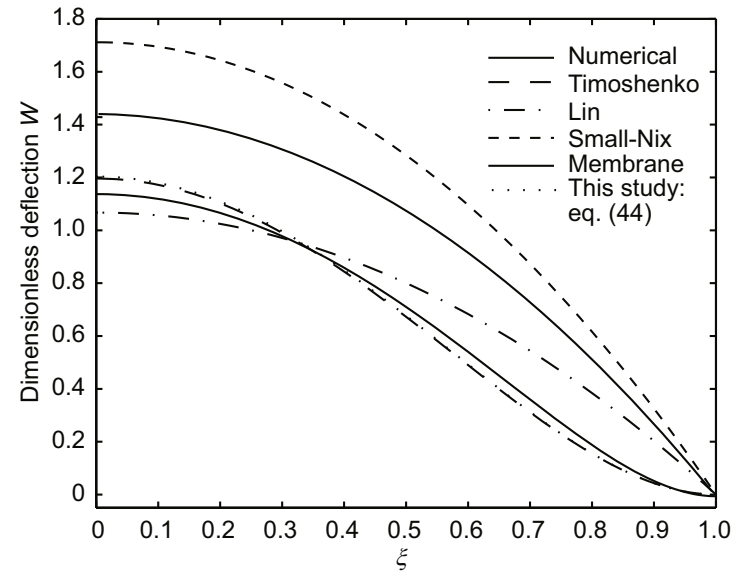

Figure 5 The plate deflections calculated by different approximate analytical solutions and numerical one when $\epsilon_{o}=1.5 \times 10^{-3}, q / E=10^{-4}$ and $a / h=20$.

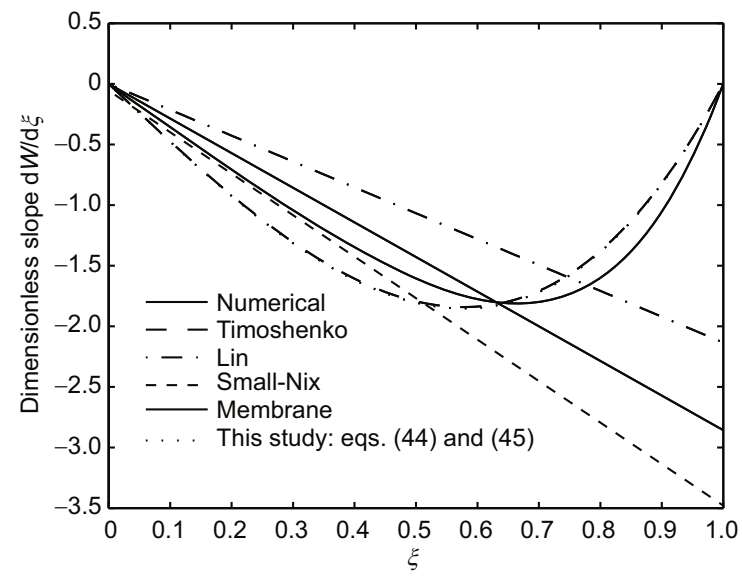

Figure 6 The plate slopes calculated by different approximate analytical solutions and numerical one when $\epsilon_{o}=1.5 \times 10^{-3}, q / E=10^{-4}$ and $a / h=20$.

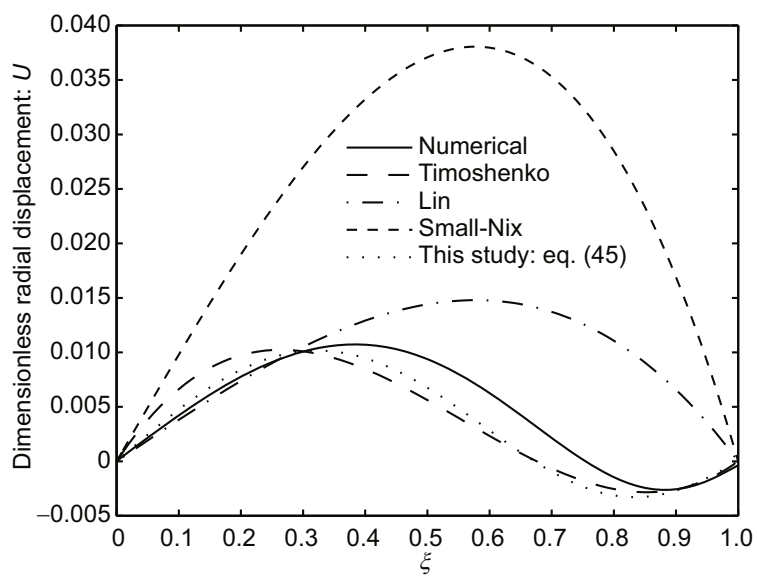

Figure 7 The plate in-plane radial displacements calculated by different approximate analytical solutions and numerical one. when $\epsilon_{o}=1.5 \times 10^{-3}$, $q / E=10^{-4}$ and $a / h=20$.

brane and Small-Nix approximations violate the clamped boundary condition of zero slope at $\xi=0$. In both Fig- ures 3 and 6, it is noticed that the slope around the edge changes relatively rapidly, which was first noted by Friderichs [51] and then systematically studied by Sheplak and Dugundji [44]. In Figure 3, the slope obtained by the numerical solution reaches the minimum of -1.426 at $\xi=0.62$ and then increases to zero at $\xi=1$; in Figure 6 , the slope by the numerical solution reaches the minimum of -1.812 at $\xi=0.67$ and then increases to zero at $\xi=1$. The slope variation around the edge in Figure 6 is more rapid than that of Figure 3. Sheplak and Dugundji [44], Tong and Huang [25] have also shown that larger $\epsilon_{o}$ or larger $q$ can make the slope variation more rapid. Figure 7 presents the radial displacement. The same scenario occurs again: the Lin's and Small-Nix approximations miss the characteristics of $U$ being positive and then negative; our approximation is still better than Timoshenko's. Compared with Figure 4, $U$ of Figure 7 is now positive in $0 \leqslant \xi \leqslant 0.76$ and negative in $0.76 \leqslant \xi \leqslant 1$. Larger transverse pressure pushes the positive zone of $U$ further towards the edge, which was also noticed by Sheplak and Dugundji [44]. These rapid change behaviors around the clamped edge are also called the boundary layer effects [26]. The plate-membrane transition behavior of deflection has been intensively studied [21, 25, 26, 44]. However, the transition behavior is demonstrated by solving eqs. (13), (14) and (15) in conjunction of the boundary conditions of eq. (17) via numerical integration [21, 26, 44] or perturbation method [25], which, by any standard, is difficult and complex. Here we present a direct and simple approach to illustrate how this transition behavior occurs. The above comparison study on the plate deflection and radial displacement shows that the Timoshenko approximation and our approximation give good approximation under relatively small or large initial in-plane strain/tension and transverse pressure. Here the Timoshenko approximation of eq. (43) is used to demonstrate the transition behavior. eq. (43) can be broken into these following two parts as for linear and cubic approximations, respectively:

$$
\left[1+\frac{3}{4}\left(\frac{a}{h}\right)^{2}(1+v) \epsilon_{o}\right] W_{o}=\frac{q a^{4}}{64 D h},
$$

and

$$
\left(0.4319+0.2411 v-0.1808 v^{2}\right) W_{o}^{3}=\frac{q a^{4}}{64 D h} .
$$

Eq. (51) is the linear part of the Timoshenko approximation, which gives $W_{o} \propto q$; eq. (52) is the nonlinear cubic term of the Timoshenko approximation, which gives $W_{o} \propto q^{1 / 3}$. Figure 8 presents a comparison study of $W_{o}$ calculated by eqs. (43), (51) and (52) when (a) $\epsilon_{o}=0$, (b) $\epsilon_{o}=1 \times 10^{-4}$ and (c) $\epsilon_{o}=1.5 \times 10^{-3}$. The $W_{o}$ calculated by eq. (43) is always smaller than those calculated by eqs. (51) and (52). When $W_{o}$ is small, eq. (51) is a better approximation of eq. (43); with the increase of $q$, the deviation enlarges and eq. (52) becomes a better one. If the error is defined as $\left(W_{o}^{\text {linear }}-W_{o}^{\text {Timoshenko }}\right) / W_{o}^{\text {Timoshenko }}$, we found that for (a) $\epsilon_{o}=0$, the error exceeds $10 \%$ at $q / E=1.7 \times 10^{-5}$ and 

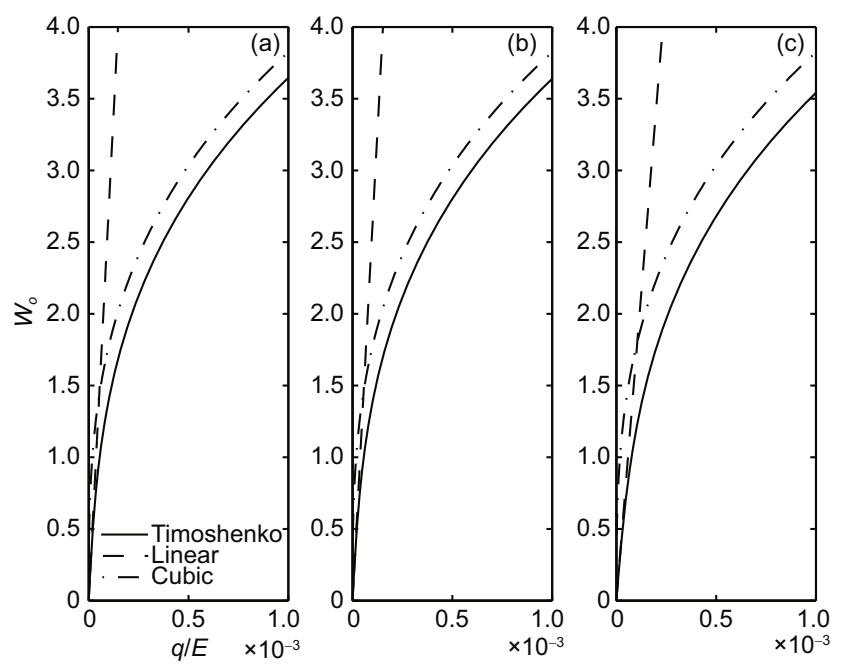

Figure 8 The plate center displacement $W_{o}$ calculated by the Timoshenko, linear and cubic approximations: (a) $\epsilon_{o}=0$, (b) $\epsilon_{o}=10^{-4}$, (c) $\epsilon_{o}=1.5 \times 10^{-3}$.

$W_{o}^{\text {Timoshenko }}=0.4263$; for (b) $\epsilon_{o}=1 \times 10^{-4}$, the error exceeds $10 \%$ at $q / E=2.2 \times 10^{-5}$ and $W_{o}^{\text {Timoshenko }}=0.5142$; for $(\mathrm{c})$ $\epsilon_{o}=1.5 \times 10^{-3}$, the error exceeds $10 \%$ at $q / E=3.9 \times 10^{-5}$ and $W_{o}^{\text {Timoshenko }}=0.6039$. With the increase of $W_{o}$, the deflection behavior sooner or later will transit from $W_{o} \propto q$ to $W_{o} \propto q^{1 / 3}$ (the so-called linear-cubic transition [14]), the presence of initial strain, or more specifically, $\epsilon_{o}(a / h)^{2}$, delays the transition, which has already been pointed out in references [25, 44].

Sheplak and Dugundji [44] defined a dimensionless parameter of $k=\sqrt{N_{o} a^{2} / D}=\sqrt{12(1+v) \epsilon_{o}}(a / h)$ to differentiate the above transition: the plate behavior dominates for $k<1$; the membrane behavior dominates for $k>20$ and $1<k<20$ is the transition zone. However, $k$ is only associated with $\epsilon_{o}$ and $a / h$, which in essence determines the stiffness. Wan [15] directly used $W_{o}$ to indicate the transition: $W_{o}<0.1$ is the bending/plate dominant zone; $W_{o}>10$ is the (nonlinear) stretching/membrane dominant zone; $0.1<W_{o}<10$ is the transition zone. It is more reasonable to use $W_{o}$ rather than $k$ to indicate such transition because $q$ also plays a role together with $\epsilon_{o}$. As seen in eq. (43) and Figure 8, when $W_{o}$ is small (for example, $W_{o}<0.1$ ), the linear term is much larger than the cubic term which only plays a secondary role of stiffening [52], $W_{o}$ varies approximately linearly with the transverse pressure. There is a subtle issue on the linearity as noticed by Komaragiri et al. [21]. The linear equation of eq. (51) consists of two parts: one is due to plate bending and the other is due to the initial strain $\epsilon_{o}$. Therefore, when $\epsilon_{o}(a / h)^{2} \ll 1$ and $W_{o}$ is small, the bending contribution is the dominant one, which is referred to as the plate behavior [21]; when $\epsilon_{o}(a / h)^{2} \gg 1$ and $W_{o}$ is small, the initial stretching contribution is the dominant one, which is referred to as the linear membrane behavior [21]. When $W_{o}$ is large (for example, $W_{o}>10$ ), the cubic term is dominant and this $W_{o} \propto q^{1 / 3}$ behavior is referred to as nonlinear membrane behavior [21]. Because $W_{o}$ is determined by both $\epsilon_{o}$ and $q$, Komaragiri et al. [21] used two dimensionless parameters: $\alpha \propto \log \left[q / E(a / h)^{2}\right]$ and $\gamma \propto \log \left(\epsilon_{o} a / h\right)$ to demarcate the three regions of plate, linear membrane and nonlinear membrane. As seen in eq. (43), $\alpha_{1}=q / E(a / h)^{4} \propto q a^{4} /(D h)$ and $\gamma_{1}=\epsilon_{o}(a / h)^{2}$ are the two dimensionless parameters directly determine the solution of $W_{o}$, which should be a more effective alternative for $\alpha$ and $\gamma$. It is also worth pointing out that all the demarcation schemes above are rather qualitative than quantitative. To quantitatively determine the contributions from different mechanisms, the analysis similar to the one presented in Figure 8 is needed.

As discussed above, some assumed displacement shapes violate the boundary conditions required by the von Kármán equations and thus become a major error source for the approximate analytical solution [14]. Now let us look at this issue from another different angle. In sect. 3, the approximate analytical solution starts with assuming the deflection shape such as eq. (18) or eq. (33). When the deflection is small, Xiao et al. [9] found that the actual deflection shape significantly deviates from the spherical/membrane shape of eq. (33) and their cosine shape. In comparison, as shown in Figures 2 and 5, the plate shape of eq. (18) shows very good approximation. Ziebart et al. [7] showed that with the increase of residual stress, the deflection shape steadily shifts towards a membrane one. In general, Sheplak and Dugundji [44], Voorthuyzen and Bergveld [8] showed that either the change of residual stress or that of transverse load $q$ can change the deflection shape, which is also reflected in Figures 2 and 5. As a result, none of the approximate analytical solution with the assumed deflection shape can accurately capture the real deflection in a wide range of $\epsilon_{o}$ and $q$. It seems that the Timoshenko approximation and our new approximation of eq. (45) are the best in the intermediate deflection range of $0.1<W_{o}<10$. Besides the deflection shape change, there is another mechanism causing the deviation. Our approximate analytical solution together with those listed in Table 1 is based on the von Kármán kinematic assumptions of eq. (1), which is for the "large" deflection of plate. For "very large" plate deflection, the Reissner plate theory [53] applies, which changes the radial strain from $\epsilon_{r}=u / r+1 / 2(\mathrm{~d} w / \mathrm{d} r)^{2}+\epsilon_{o}$ of eq. (1) to $\epsilon_{r}=u / r+1 / 2\left[(\mathrm{~d} u / \mathrm{d} r)^{2}+(\mathrm{d} w / \mathrm{d} r)^{2}\right]+\epsilon_{o}$. This additional strain contribution of $1 / 2(\mathrm{~d} u / \mathrm{d} r)^{2}$ changes the energy expressions of eqs. (3) and (4) and thus the differential governing equation set of eqs. (6) and (7) and that of eqs. (13), (14) and (15). With the increase of deflection, the contribution of $1 / 2(\mathrm{~d} u / \mathrm{d} r)^{2}$ becomes more and more important, which makes the above equations less and less accurate. In Figures 2 and 5, it is noticed that $W_{o} \propto 1$, which is the intermediate range of deflection [15] and the case of large deflection. A lot of researchers have used the von Kármán equations to compute the deflection case of $W_{o}>50$. The caution should be taken on those results though there is no clear boundary between the "large" and "very large" deflections. 


\section{Conclusion}

A fourth order and a second governing equations on the large deflection of plates are derived by the principle of virtual work, which also gives an extra boundary condition of the zero radial displacement at the center. We show that one of the previous boundary conditions $\left(S_{r}(0)=S_{t}(0)\right)$ is unnecessary. On the other hand, the zero radial displacement at the center must be enforced. Otherwise, it will cause the singularity problem. The approximate analytical forms are derived with the Poisson's ratio as a variable and compared with the numerical integration results. The plate shape of deflection and its corresponding radial displacement form achieve a better approximation than that of assuming the membrane shape deflection in the intermediate range of plate deflection. The membrane shape violates the clamped boundary conditions of zero slope at the edge and also misses the radial displacement characteristics of being positive and then negative along the span. A new approximation method is proposed, which makes the radial displacement satisfy the governing equation with the assumed deflection shape of plate. The new approximation on the radial displacement is better than Timoshenko's. Through the nondimensionalization scheme, it is found that $q / E(a / h)^{4}$ and $\epsilon_{o}(a / h)^{2}$ are the two dimensionless parameters determining the plate deflection behavior. The Timoshenko approximation or our new approximation offers a framework, which can give a much simpler and more direct explanation on the plate-membrane transition behavior.

This work was supported by the National Natural Science Foundation of China (Grant No. 11372321).

1 M. Small, and W. D. Nix, J. Mater. Res. 7, 1553 (1992).

2 M. Small, B. J. Daniels, B. M. Clemens, and W. D. Nix, J. Mater. Res. 9, 25 (1994).

3 J. J. Vlassak, and W. D. Nix, J. Mater. Res. 7, 3242 (1992).

4 Y. Xiang, X. Chen, and J. J. Vlassak, J. Mater. Res. 20, 2360 (2005).

5 P. Lin, The In-situ Measurement of Mechanical Properties of Multi Layer Coatings, Dissertation for the Doctoral Degree (MIT, Boston, 1990).

6 T. Y. Zhang, Y. Su, J. Qian, M. H. Zhao, and L. Q. Chen, Acta Mater. 48, 2843 (2000)

7 V. Ziebart, O. Paul, U. Münch, J. Schwizer, and H. Baltes, J. Microelectromech. Syst. 7, 320 (1998).

8 J. A. Voorthuyzen, and P. Bergveld, Sens. Actuators 6, 201 (1984).

9 Z. Xiao, O. Engström, and N. Vidovic, Sens. Actuators A 58, 99 (1997).

10 M. K. Tripp, C. Stampfer, D. C. Miller, T. Helbling, C. F. Herrmann, C. Hierold, K. Gall, S. M. George, and V. M. Bright, Sens. Actuators A 130-131, 419 (2006).

11 J. S. Hsu, L. P. Chao, J. H. Jhong, T. F. Chen, and W. C. Tsai, Opt. Lasers Engr. 48, 354 (2010).

12 T. Süss, P. Braeuninger-Weimer, and C. Hierold, Sens. Actuators A 212, 159 (2014).

13 J. G. Williams, Int. J. Fracture 87, 265 (1997).
14 K. T. Wan, S. Guo, and D. A. Dillard, Thin Solid Films 425, 150 (2003).

15 K. T. Wan, J. Appl. Mech. 69, 110 (2002).

16 K. T. Wan, and Y. W. Mai, Acta Metall. Mater. 43, 4109 (1995).

17 S. P. Koenig, N. G. Boddeti, M. L. Dunn, and J. S. Bunch, Nat. Nanotech. 6, 543 (2011).

18 Y. Zhang, J. Mater. Sci. 43, 88 (2008).

19 Y. Zhang, J. Phys. D-Appl. Phys. 43, 1188 (2007).

20 Y. Zhang, J. Micromech. Microengr. 17, 753 (2007).

21 U. Komaragiri, M. R. Begley, and J. G. Simmonds, J. Appl. Mech. 72, 203 (2005).

22 M. R. Begley, and T. J. Mackin, J. Mech. Phys. Solids 52, 2005 (2004).

23 B. F. Ju, Y. Ju, M. Saka, K. K. Liu, and K. T. Wan, Int. J. Mech. Sci. 47, 319 (2005).

24 S. P. Timoshenko, and S. Woinowsky-Krieger, Theory of Plates and Shells (2nd ed) (McGraw-Hill, New York, 1959).

25 P. Tong, and W. Huang, J. Appl. Mech. 69, 785 (2002).

26 Y. H. Su, K. S. Chen, D. C. Roberts, and S. M. Spearing, J. Micromech. Microengr. 11, 645 (2001).

27 Y. Zhang, and K. D. Murphy, Acta. Mech. Sin. 28, 1374 (2012).

28 M. H. Zhao, F. Yang, and T. Y. Zhang, Mech. Mater. 39, 881 (2007).

29 H. X. Mei, C. M. Landis, and R. Huang, Mech. Mater. 43, 627 (2011).

30 K. Yue, W. Gao, R. Huang, and K. M. Liechti, J. Appl. Phys. 112, 083512 (2012).

31 T. Georgiou, L. Britnell, P. Blake, R. V. Gorbachev, A. Gholinia, A. K. Geim, C. Casiraghi, and K. S. Novoselov, Appl. Phys. Lett. 99, 093103 (2011).

32 A. Boudaoud, P. Patricio, Y. Couder, and M. B. Amar, Nature 407, 718 (2000).

33 A. Nádai, Die Elastischen Platten (Springer, Berlin, 1925).

34 S. Way, Trans. ASME 56, 627 (1934).

35 K. Federhofer, Forschungsarb 7, 148 (1936).

36 A. McPherson, W. Ramberg, and S. Levy, Normal Pressure Tests of Circular Plates with Clamped Edges, NACA Report No. 744 (1942).

37 P. Lin, and S. D. Senturia, Mater. Res. Soc. Symp. Proc. 188, 41 (1990).

38 W. K. Schomburg, Introduction to Microsystem Design (Springer, Berlin Heidelberg, 2011).

39 H. Hencky, Z. Math. Phys. 63, 311 (1915).

40 A. N. Gent, and L. H. Lewandowski, J. Appl. Polym. Sci. 33, 1567 (1987).

41 J. W. Beams, Mechanical properties of thin films of gold and silver, in Structures and Properties of Thin Films, edited by C. A. Neugebauer, J. B. Newkirk, D. A. Verilyea (John Wiley and Sons, New York, 1959), pp. 183-192.

42 M. G. Allen, and S. D. Senturia, J. Adhes. 25, 303 (1988).

43 J. Sizemore, R. J. Hohlfelder, J. J. Vlassak, and W. D. Nix, Mater. Res. Soc. Symp. Proc. 383, 197 (1995).

44 M. Sheplak, and J. Dugundji, J. Appl. Mech. 65, 107 (1998).

45 X. T. He, Q. Chen, J. Y. Sun, and Z. L. Zheng, Int. J. Mech. Sci. 62, 103 (2012).

46 W. Z. Chien, Chin. J. Phys. 7, 102 (1947).

47 W. Z. Chien, and K.Y. Yeh, Chin. J. Phys. 10, 209 (1954).

48 G. A. Korn, and T. M. Korn, Mathematical Handbook for Scientist and Engineers (2nd ed) (McGraw-Hill Book Company, New York, 1968).

49 X. J. Zheng, and Y. H. Zhou, Acta Mech. Sin. 6, 69 (1990).

50 W. H. Press, S. A. Teukolsky, W. T. Vetterling, and B. P. Flannery, Numerical Recipes in Fortran (2nd ed) (Cambridge University Press, Cambridge, 1992).

51 K. O. Friderichs, Proc. Symposia. Appl. Math. 1, 188 (1949).

52 Y. Zhang, Y. Liu, and K. D. Murphy, Acta. Mech. Sin. 28, 190 (2012).

53 E. Reissner, Proc. Symposia. Appl. Math. 1, 213 (1949). 